\title{
Idiopathic pulmonary artery aneurysm: is it really idiopathic?
}

\author{
Şafak Alpat ${ }^{1}$, Tanju Ylldon ${ }^{1}$, Dursun Alehan² ${ }^{2}$ Mustafa Yllmaz ${ }^{1}$ \\ ${ }^{1}$ Department of Cardiovascular Surgery, Medical Faculty of Hacettepe University, Ankara, Turkey \\ ${ }^{2}$ Department of Paediatric Cardiology, Medical Faculty of Hacettepe University, Ankara, Turkey \\ Received: December 08, 2015 Accepted: September 07, 2016 Published online: January 11, 2017
}

\section{ABSTRACT}

Pulmonary artery aneurysms are rare cardiac abnormalities. Although various factors have been proposed, their etiology and pathophysiology are not well-understood. Herein, we report our surgical approach for a symptomatic pulmonary artery aneurysm in an 11-year-old girl, in whom we detected an intraluminal structure during surgery.

Keywords: Idiopathic; pulmonary artery aneurysm; turbulent flow.

A pulmonary artery $(\mathrm{PA})$ aneurysm is a rare lesion with an approximate incidence of $1 / 14,000$ in autopsies. ${ }^{[1]}$ In the literature, various etiologic causes have been previously reported, of which idiopathic PA aneurysm being the most common. ${ }^{[1]}$ The main complaints of a PA aneurysm include shortness of breath, cough, and chest pain. ${ }^{[2]}$ There is no guideline for surgical intervention in PA aneurysm and management still remains controversial. In general, early surgical intervention is recommended for symptomatic cases and large PA aneurysm sizes to avoid possible fatal ruptures. ${ }^{[2]}$ Herein, we report a rare case of a PA aneurysm and its successful management with surgical intervention. Besides, we propose a new hypothesis for a cause of a PA aneurysm in our case.

\section{CASE REPORT}

An 11-year-old female patient was referred to our institution with a complaint of hoarseness and shortness of breath (New York Heart Association [NYHA] Class II) which were progressively increasing with the past six months. There was no history of cough, orthopnea, hemoptysis, fever, chest pain, syncope or trauma. Physical examination findings were completely normal, except grade III/IV harsh systolic murmur over the pulmonic area. Electrocardiography showed sinus rhythm. Chest X-ray demonstrated an aneurysmal main PA segment without cardiomegaly and with normal lung fields. Transthoracic echocardiography (TTE) revealed a main PA aneurysm without any other cardiac abnormalities. The patient was also examined for connective tissue disorders, which produced negative results. Cardiac magnetic resonance imaging (MRI) was done to further evaluate the aneurysm and showed a lesion with $3 \mathrm{~cm}$ fusiform dilatation of the main PA towards the left PA above the pulmonary valve. Distal main PA and branch PAs were normal in size (Figure 1). During catheterization, maximal diameter was $38 \mathrm{~mm}$ (X-score: 4.9) and the main PA pressure was $26 \mathrm{mmHg}$ (Figure 2). Interestingly, the filling of the right PA with contrast agent was delayed and relatively slower than that of left PA. However, it was initially considered an artefact, as there was no accompanying cardiac or vascular abnormality, as assessed by TTE, MRI and angiography.

As the patient was symptomatic with a main PA $\mathrm{Z}$-score of 4.9 , we performed elective surgery. A midline sternotomy was performed. The main PA was aneurysmatic towards the left PA. Cardiopulmonary bypass under mild hypothermia was initiated. Vertical pulmonary arteriotomy revealed no thrombi or vegetations. The right ventricular outflow tract, pulmonary valve, and branch PAs were normal. However, an intraluminal membranous flap-like structure which partially obstructed the right PA origin was detected. The membrane and aneurysmatic arterial segment were resected. Redundant arterial wall was reconstructed, according to an appropriate Hegar

Corresponding author: Şafak Alpat, MD., PhD. Hacettepe Üniversitesi Tip Fakültesi Kalp ve Damar Cerrahisi Anabilim Dalı, 06100 Sihhiye, Ankara, Turkey. Tel: +90 537 - 2419710 e-mail: salpat@hacettepe.edu.tr 


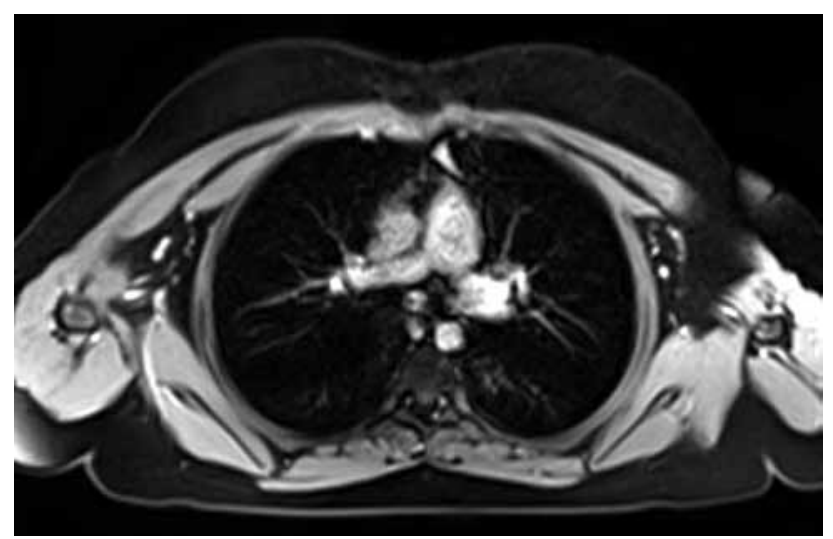

Figure 1. A coronal section of cardiac magnetic resonance imaging showing fusiform dilatation of main pulmonary artery towards left pulmonary artery and normal-size branch pulmonary arteries.

dilator size. Postoperative course was uneventful. Prior to discharge, repeated echocardiography showed no gradient across the pulmonary valve and the patient was, then, was discharged in the fifth postoperative day. Histopathological examination of the arterial wall revealed a lost smooth muscle layer which was replaced by diffuse mural fibrosis. Elastic fibers were disrupted and fragmented. There were no vasculopathic changes in the adventitia (Figure 3).

\section{DISCUSSION}

Aneurysms of the main PA are rare clinical entities with an unknown etiology and pathogenesis. ${ }^{[1]}$ Almost half of the cases have accompanying congenital heart disease, the most common being patent ductus arteriosus. ${ }^{[3]}$ Behçet's and Marfan syndromes, injuries, pulmonary hypertension, infections, and idiopathic factors are the other common underlying causes of these aneurysms. ${ }^{[3]}$ The diagnosis should be based on TTE and cardiac catheterization findings to ascertain the extent of the aneurysm and rule out accompanying intracardiac pathologies. ${ }^{[2,3]}$ In addition, MRI is an essential diagnostic tool, as it is noninvasive and optimal for the detection of intimal flaps. ${ }^{[2,3]}$ Furthermore, in case of suspected connective tissue disorders, rheumatologic panel should be tested. Our case suffered from dyspnea and hoarseness. The first was reported as the most common symptom of patients with a PA aneurysm, while the latter is a rare symptom. In a PA aneurysm, hoarseness resulted from stretching of the recurrent laryngeal nerve. ${ }^{[2,3]}$ For diagnostic workup, several imaging modalities were used, including TTE, catheterization, and MRI; however, none showed an accompanying abnormality. Furthermore, rheumatologic panel was completely normal.

The clinical course of large aneurysms is unpredictable. According to the Laplace's law, wall tension is directly proportional to the radius of the vessel and intraluminal pressure, whereas it is inversely related to the wall thickness. ${ }^{[4]}$ Thus, higher intraluminal pressures with thinning of the vessel wall lead to an increased risk of dilatation and rupture. ${ }^{[4]}$ Also, it may cause massive hemoptysis. ${ }^{[2]}$ In the literature, there is still a debate on the optimal size of the main
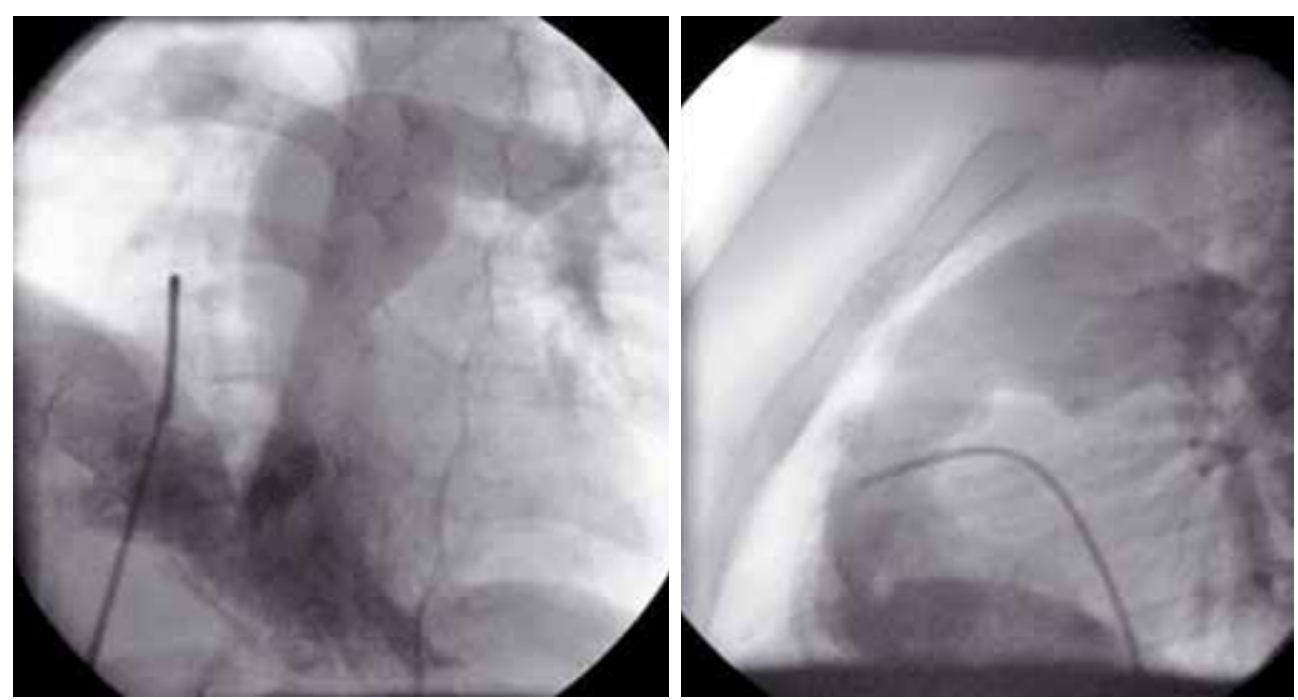

Figure 2. Anteroposterior and left lateral views during cardiac catheterization showing a main pulmonary artery aneurysm. 

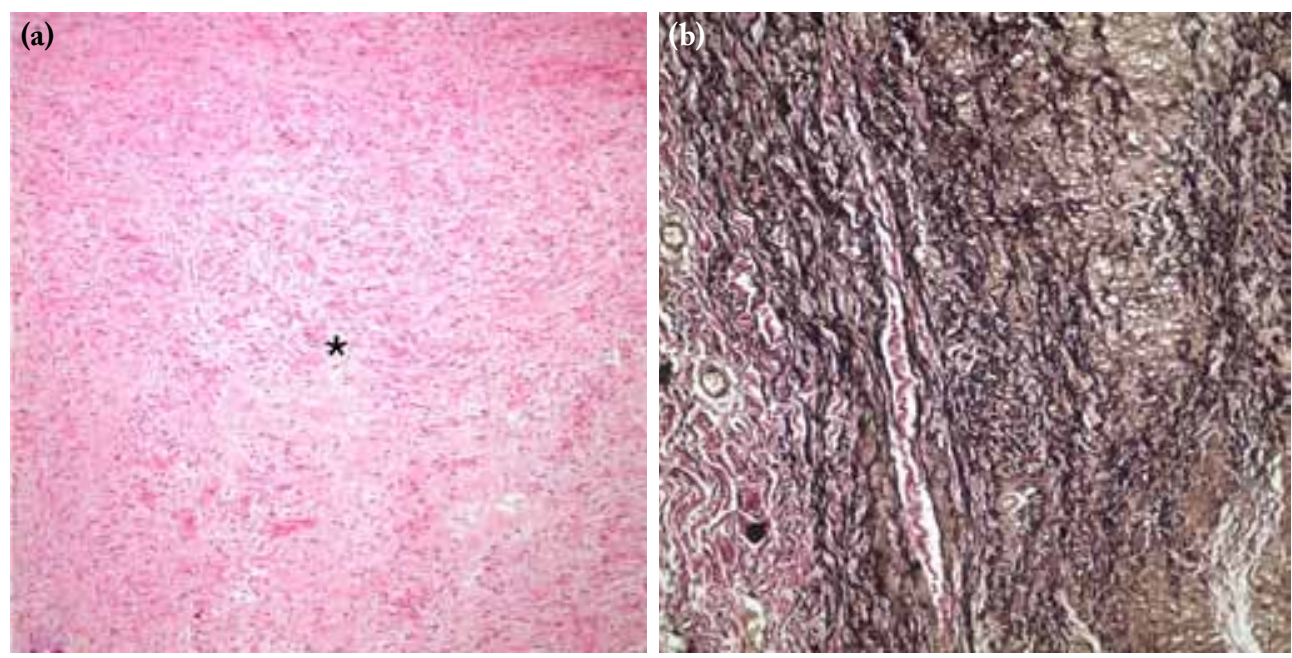

Figure 3. Histopathological examination of the arterial wall. (a) Loss of smooth muscle layer $\left.{ }^{*}\right)$ (H-E x 10). (b) Disrupted and fragmented elastic fibers (black stained) (Verhoeff's stain x 20).

PA aneurysms for surgical indication. On the other hand, it is commonly accepted that symptomatic aneurysms should be surgically treated to prevent the risk of rupture. ${ }^{[2]}$ As there is no clear cut-off level of the aneurysm size for surgery, we preferred using $\mathrm{X}$-score (4.9) as a surgical indication. Therefore, we believe that using $\mathrm{X}$-scores may be crucial in timing of surgery.

Furthermore, various surgical techniques have been described to date, including aneurysm plication, pericardial patch reconstruction, and graft interposition. ${ }^{[3]}$ Review of case series revealed that these techniques were equally effective. ${ }^{[3]}$ In our case, we used arterial resection with reduction arterioplasty to preserve the intact pulmonary valve function and integrity of the branch PAs.

On the other hand, an interesting finding in our case was the existence of an intraluminal membranous flap-like structure which partially obstructed the right PA origin. Slow, late-phase filling of the right PA during preoperative angiography was most likely related to this structure. Unfortunately, preoperative MRI did not show any flap-like structure in PAs. We hypothesize that membrane around the origin of the right PA led to turbulent flow which was injuring the internal wall of left PA. Consistent with this hypothesis, we noticed that the main PA aneurysm was leaning towards the left PA. Previous reports showed that different flow dynamics and disturbed flow conditions might damage the endothelium, thereby, offering the initial step to the degeneration of the arterial wall. ${ }^{[4]}$ Consequently, they may cause aneurysm formation, as turbulence significantly increases the pressure and fluid shear stress over the arterial wall. Histopathological findings also supported our hypothesis. In previously reported cases, normal histology was reported. ${ }^{[1,5]}$ However, we found a complete loss of the muscle layer and replacement fibrosis without accompanying adventitial alterations. Therefore, these alterations may have occurred as a result of turbulent flow caused by the membranous structure.

In conclusion, despite numerous etiologic factors of pulmonary artery aneurysms have been identified, surgeons should keep in mind that some biomechanical causes may be the underlying factors of aneurysms in so-called idiopathic cases.

\section{Declaration of conflicting interests}

The authors declared no conflicts of interest with respect to the authorship and/or publication of this article.

\section{Funding}

The authors received no financial support for the research and/or authorship of this article.

\section{REFERENCES}

1. Seguchi M, Wada H, Sakakura K, Kubo N, Ikeda N, Sugawara $\mathrm{Y}$, et al. Idiopathic pulmonary artery aneurysm. Circulation 2011;124:e369-70.

2. Nair KK, Cobanoglu AM. Idiopathic main pulmonary 
artery aneurysm. Ann Thorac Surg 2001;71:1688-90.

3. Deb SJ, Zehr KJ, Shields RC. Idiopathic pulmonary artery aneurysm. Ann Thorac Surg 2005;80:1500-2.

4. Lasheras JC. The biomechanics of arterial aneurysms.
Annu Rev Fluid Mech 2007;39:293-319.

5. Agarwal S, Chowdhury UK, Saxena A, Ray R, Sharma S, Airan B. Isolated idiopathic pulmonary artery aneurysm. Asian Cardiovasc Thorac Ann 2002;10:167-9. 